\title{
KREDITE
}

\section{Offene Kommunikation als Basis}

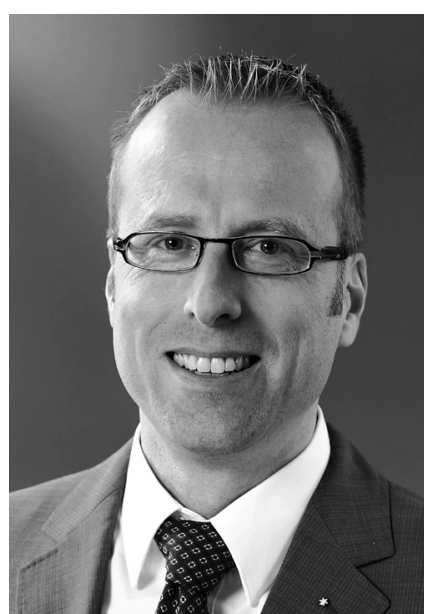

VON OLIVER LUCKNER Oliver Luckner ist Bankkaufmann und Absolvent der Frankfurt School of Finance (Bankakademie). Seit drei Jahren ist er Generalbevollmächtigter der Bank für Sozialwirtschaft AG und als solcher zuständig für die Marktunterstützung und Vertriebssteuerung der Bank. Von 2000 bis 2010 war der Kreditspezialist für Einrichtungen und Unternehmen der Sozialwirtschaft als Direktor Norddeutschland zuständig für die Kundenbetreuung und den Vertrieb der Bank für Sozialwirtschaft AG an den Standorten Hamburg und Hannover.

www.sozialbank.de

\author{
Da die öffentliche Finanzierung sozialer Angebote \\ vielfach stagniert oder zurückgeht, wächst das \\ Kreditvolumen im Sozial- und Gesundheitswesen \\ seit Jahren stetig. Sowohl die Banken als auch die \\ Sozialunternehmen müssen sich langfristig und \\ gemeinsam auf diese Situation einstellen.
}

Die Sozial- und Gesundheitswirtschaft wird auch in den nächsten Jahren ein Wachstumsmarkt bleiben. Die demographische Entwicklung und eine wachsende Vielfalt von Bedarfslagen und Kundenerwartungen werden eine weiter steigende Nachfrage nach Leistungen in den Branchen Altenhilfe, Behindertenhilfe, Kinder- und Jugendhilfe, Gesundheitswesen etc. mit sich bringen.

Gleichzeitig werden sowohl die finanziellen als auch die personellen Ressourcen der Unternehmen knapper. Um die damit verbundenen Herausforderungen zu bewältigen, ist Innovationsfähigkeit gefordert: Angebotsstrukturen und Leistungsprozesse müssen weiterentwickelt werden. Die Unternehmen sind gefordert, sich durch nachfragegerechte Angebote und tragfähige Geschäftsmodelle zukunftsfähig aufzustellen. Das fordern nicht nur die Kunden und Nutzer; zunehmend werden auch die politischen Rahmenbedingungen neu gesetzt. Das Konzeptionieren und Etablieren neuer Versorgungsformen, die Weiterentwicklung bestehender Angebote und Leistungen, vielfältige Varianten von Kooperationen und $\mathrm{Fu}$ sionen sowie eine zunehmende sektorübergreifende Vernetzung sind nur einige Konsequenzen daraus.

Die damit verbundenen Maßnahmen erfordern Investitionen. Da die öffentlich regulierte Finanzierung an vielen Stellen nicht mehr greift, wächst das Kreditvolumen im Sozial- und Gesundheitswesen seit Jahren stetig. Parallel dazu hat die Zahl der Insolvenzen in der Sozialwirtschaft zugenommen. Für Kapitalgeber bedeutet das: Eine genaue Kenntnis der Finanzierungsbedingungen in den einzelnen Branchen der Sozialwirtschaft ist ebenso unabdingbar wie eine Einzelfallprüfung des jeweiligen $\mathrm{Fi}$ nanzierungsvorhabens.

Bei der Entscheidung über eine Kreditvergabe spiegelt sich dies u. a. darin, dass inzwischen die Frage nach dem Geschäftsmodell des Unternehmens zum zentralen Aspekt geworden ist: Warum muss es Ihr Unternehmen geben? Was macht es nachhaltig wettbewerbsfähig? Wie sieht Ihre Unternehmensstrategie aus? Welche baulichen, personellen und betriebsorganisatorischen Voraussetzungen bringen Sie mit? Wie passt Ihr Angebot in die örtliche Versorgungsstruktur? Wie stark sind Sie mit anderen Akteuren vernetzt? Das sind nur einige Fragen, die zu stellen sind.

Die Marktfähigkeit eines Geschäftsmodells kann beispielsweise für Pflegeimmobilien anhand einer Standort- und Wettbewerbsanalyse geprüft werden. Diese richtet ihren Fokus auf drei Schwerpunkte: Zunächst wird der Status des Betreibers betrachtet und analysiert, wie kompatibel sich das geplante Vorhaben zu der unternehmerischen Gesamtstrategie darstellt. Im zweiten Schritt werden das Pflegekonzept und das räumliche Angebot unter die Lupe genommen. Entspricht das Pflegekonzept den örtlichen Nachfragepräferenzen? Wird die Raumpla- 
nung den Anforderungen gerecht, die aus dem Pflegekonzept resultieren? Anschließend wird das Angebot mit denen der bestehenden Wettbewerber vor Ort verglichen und bewertet. Eine zentrale Rolle spielen bei der gesamten Analyse die Bedingungen im Einzugsgebiet (Altersstruktur, Morbidität, Einkommenssituation etc.). Dazu gehört auch die Frage, ob genügend Fachkräfte zur Verfügung stehen.

\section{Drei Finanzkennzahlen entscheiden über Ausfallwahrscheinlichkeit}

Ebenso wichtig wie das Geschäftsmodell ist für eine Bank die Bonität. Denn sie gibt Aufschluss über die Fähigkeit, finanzielle Verpflichtungen vollständig und fristgerecht erfüllen zu können. Bei der Ermittlung der Bonität eines potenziellen Kreditnehmers geht es darum, die Ausfallwahrscheinlichkeit des Kunden festzustellen.

Als Spezialkreditinstitut für die Sozial- und Gesundheitswirtschaft setzt die Bank für Sozialwirtschaft dazu ein Ratingsystem ein, das die Rahmenbedingungen sozialwirtschaftlicher Unternehmen berücksichtigt. Für die Beurteilung der Ausfallwahrscheinlichkeit haben sich in diesem Ratingsystem drei Finanzkennzahlen als besonders aussagekräftig erwiesen: die erweiterte Eigenkapitalquote, der Liquiditätsstatus und die Umsatzrentabilität. Darüber hinaus werden zahlreiche »soft facts « abgefragt, also Informationen zu den Rahmenbedingungen des Unternehmens und des Marktes.

Das Rating basiert überwiegend auf vergangenheitsbezogenen Daten. Ein Finanzierungsvorhaben jedoch ist in die Zukunft gerichtet. Entscheidend ist die künftige Kapitaldienstfähigkeit des Unternehmens. Daher ist für eine positive Finanzierungsentscheidung eine belastbare Planungsrechnung über etwa fünf Jahre erforderlich. Diese muss plausibel darlegen, ob der Kreditnehmer Zinsen und Tilgung bezahlen kann.

Dafür ist es erforderlich, eine integrierte mittelfristige Planungsrechnung zu erstellen, die aus einem Investitionsund Finanzplan, Plan-Gewinn- und Verlustrechnungen, Plan-Bilanzen sowie einer Plan-Cashflow-Rechnung besteht. Für die finanzierende Bank steht der Cashflow im Mittelpunkt. Welche

\section{Was versteht man eigentliche unter Cashflow?}

Der Cashflow definiert sich als positiver Zahlungsmittelüberschuss der wirtschaftlichen Tätigkeit. Dieser Überschuss bezieht sich dabei auf Erträge und Aufwendungen, die nicht nur erfolgswirksam, sondern auch zahlungswirksam sind, also in derselben Periode zu Einzahlungen oder Auszahlungen führen. Der Cashflow in der Bilanzanalyse versucht die wirklichen Zahlungsströme abzubilden, denn im bilanziellen Ergebnis eines Unternehmens sind eine

Auswirkungen haben die künftigen innerbetrieblichen Voraussetzungen und die Entwicklung der Rahmenbedingungen auf den künftigen Cashflow? Die Antwort auf diese Frage ist für das Unternehmen ebenso wichtig wie für den Kapitalgeber.

Auch insgesamt gesehen verlaufen die Interessen von Unternehmen und Kapitalgeber parallel: Nur mit einer zukunftsfähigen Strategie und einer soliden Planung wird ein Unternehmen langfristig am Markt bestehen können. Und nur dann wird es seinen finanziellen Verpflichtungen nachkommen können. Wenn also die finanzierende Bank eine möglichst genaue Risikoeinschätzung vornimmt, ist das im gemeinsamen Interesse. Sind die Risiken zu hoch, macht eine Kreditvergabe keinen Sinn - für keinen von beiden. Sind die Risiken hoch, aber noch tragfähig, wird sich das in schlechteren Konditionen niederschlagen. Mit dem Inkrafttreten von »Basel III « - einer weiteren Verschärfung der Eigenkapitalregeln für Banken - wird sich die Bedeutung des Kreditrisikos für die Konditionengestaltung noch erhöhen. Positiv gesehen: Wirtschaftlich gut aufgestellte Sozialunternehmen werden dann für Kreditgeber noch attraktiver sein als heute.

$\mathrm{Zu}$ einer realistischen Einschätzung eins Finanzierungsvorhabens gehört auch eine Bewertung der Sicherheiten, die der Kreditnehmer stellt. Banküblich ist beispielsweise die Bewertung der zu finanzierenden Immobilie. Bei der Finanzierung von Sozialimmobilien ist allerdings zu beachten, dass diese nur bedingt verwertbar sind. Pflegeheime, Krankenhäuser und Behindertenwerkstätten sind nur als Betriebe interessant, als Immobilien kaum. Sie sind nur so viel wert wie der Ertrag, der dort erwirtschaftet wird. Aus diesem Grund
Vielzahl von Faktoren wie Abschreibungen und Rückstellungen enthalten, die sich nicht auf den realen Zahlungsfluss auswirken. Ein positiver Cashflow versetzt ein Unternehmen in die Lage, aus den Umsatzprozessen heraus Kredite ordnungsgemäß zu tilgen oder neue Anlageinvestitionen zu tätigen.

Quelle: wikipedia.org

ist nicht das Gebäude selbst, sondern seine Funktionalität zu bewerten. Für Sozialimmobilien sollten daher spezielle Standards für die Ermittlung des Beleihungswertes angewendet werden, wie sie etwa »HypZert-Gutachten " bieten. Anders ist dies beispielsweise bei Angeboten des Wohnens mit ambulanter Betreuung und Pflege. Wenn sie in Wohngebäuden untergebracht sind, verfügen sie über bessere Drittverwendungsmöglichkeiten.

Insbesondere die Finanzierung einer Sozialimmobilie ist eine langfristige Angelegenheit. Daher darf die enge $\mathrm{Zu}-$ sammenarbeit zwischen Unternehmen und Bank nicht mit der Kreditvergabe enden. Eine langfristig erfolgreiche Geschäftsbeziehung wird von einer offenen Kommunikation zwischen Kreditnehmer und Bank getragen. Dazu gehören regelmäßige Informationen über das operative Geschäft sowie über die aktuelle Ertragslage und die Liquiditätssituation des Unternehmens. Kritische Entwicklungen können insbesondere dann frühzeitig erkannt werden, wenn ein kontinuierlicher Abgleich der Ist-Zahlen mit den PlanZahlen erfolgt. Dies ist bei der Bank für Sozialwirtschaft über ein »Frühindikatorensystem « möglich. Dieses Instrument vergleicht quartalsweise Ist- und Plan-Werte des Kreditnehmers. Sollte eine kritische Situation eintreten, kann die Bank ihren Kunden aktiv bei der Krisenbewältigung unterstützen, beispielsweise bei der Beseitigung von Liquiditätsengpässen.

Ziel sollte immer eine partnerschaftliche Zusammenarbeit und ein vertrauensvolles Verhältnis zwischen Kreditnehmer und finanzierender Bank sein. 